\title{
Water Quality Index for measuring drinking water quality in rural Bangladesh: a cross- sectional study
}

\author{
Tahera Akter ${ }^{1}$, Fatema Tuz Jhohura ${ }^{1}$, Fahmida Akter ${ }^{1}$, Tridib Roy Chowdhury', Sabuj Kanti Mistry', Digbijoy Dey ${ }^{3}$, \\ Milan Kanti Barua ${ }^{3}$, Md Akramul Islam $^{2,3}$ and Mahfuzar Rahman ${ }^{1 *}$
}

\begin{abstract}
Background: Public health is at risk due to chemical contaminants in drinking water which may have immediate health consequences. Drinking water sources are susceptible to pollutants depending on geological conditions and agricultural, industrial, and other man-made activities. Ensuring the safety of drinking water is, therefore, a growing problem. To assess drinking water quality, we measured multiple chemical parameters in drinking water samples from across Bangladesh with the aim of improving public health interventions.

Methods: In this cross-sectional study conducted in 24 randomly selected upazilas, arsenic was measured in drinking water in the field using an arsenic testing kit and a sub-sample was validated in the laboratory. Water samples were collected to test water $\mathrm{pH}$ in the laboratory as well as a sub-sample of collected drinking water was tested for water $\mathrm{pH}$ using a portable $\mathrm{pH}$ meter. For laboratory testing of other chemical parameters, iron, manganese, and salinity, drinking water samples were collected from 12 out of 24 upazilas.

Results: Drinking water at sample sites was slightly alkaline ( $\mathrm{pH} 7.4 \pm 0.4)$ but within acceptable limits. Manganese concentrations varied from 0.1 to $5.5 \mathrm{mg} / \mathrm{L}$ with a median value of $0.2 \mathrm{mg} / \mathrm{L}$. The median iron concentrations in water exceeded WHO standards $(0.3 \mathrm{mg} / \mathrm{L})$ at most of the sample sites and exceeded Bangladesh standards $(1.0 \mathrm{mg} / \mathrm{L})$ at a few sample sites. Salinity was relatively higher in coastal districts. After laboratory confirmation, arsenic concentrations were found higher in Shibchar (Madaripur) and Alfadanga (Faridpur) compared to other sample sites exceeding WHO standard (0.01 mg/L). Of the total sampling sites, $33 \%$ had good-quality water for drinking based on the Water Quality Index (WQI). However, the majority of the households (67\%) used poor-quality drinking water.
\end{abstract}

Conclusions: Higher values of iron, manganese, and arsenic reduced drinking water quality. Awareness raising on chemical contents in drinking water at household level is required to improve public health.

Keywords: Water quality index, Chemical parameters, WASH program, BRAC, Bangladesh

\section{Background}

Quality of drinking water indicates water acceptability for human consumption. Water quality depends on water composition influenced by natural process and human activities. Water quality is characterized on the basis of water parameters (physical, chemical, and microbiological), and human health is at risk if values exceed acceptable limits [1-3]. Various agencies such as the

\footnotetext{
* Correspondence: mahfuzar.rahman@brac.net

${ }^{1}$ BRAC Research and Evaluation Division, BRAC Centre, 75 Mohakhali, Dhaka

1212, Bangladesh

Full list of author information is available at the end of the article
}

World Health Organization (WHO) and Centers for Disease Control (CDC) set exposure standards or safe limits of chemical contaminants in drinking water. A common perception about water is that clean water is good-quality water indicating knowledge gap about the presence of these substances in water. Ensuring availability and sustainable management of good-quality water is set as one of the Sustainable Development Goals (SDGs) and is a challenge for policy makers and Water, Sanitation and Hygiene (WASH) practitioners, particularly in the face of changing climatic conditions, increasing populations, poverty, and the negative effects of human development. 
Water Quality Index (WQI) is considered as the most effective method of measuring water quality. A number of water quality parameters are included in a mathematical equation to rate water quality, determining the suitability of water for drinking [4]. The index was first developed by Horton in 1965 to measure water quality by using 10 most regularly used water parameters. The method was subsequently modified by different experts. These indices used water quality parameters which vary by number and types. The weights in each parameter are based on its respective standards, and the assigned weight indicates the parameter's significance and impacts on the index. A usual WQI method follows three steps which include (1) selection of parameters, (2) determination of quality function for each parameter, and (3) aggregation through mathematical equation [5]. The index provides a single number that represents overall water quality at a certain location and time based on some water parameters. The index enables comparison between different sampling sites. WQI simplifies a complex dataset into easily understandable and usable information. The water quality classification system used in the WQI denotes how suitable water is for drinking. The single-value output of this index, derived from several parameters, provides important information about water quality that is easily interpretable, even by lay people [6]. In a resource-poor country like Bangladesh where ensuring availability and sustainable management of water is one of the challenging areas towards development. The present study embraced weighted arithmetic WQI method to deliver water quality information to WASH practitioners. One of the merits of this method is that a less number of parameters are required to compare water quality for certain use [5].

The WASH program of the Bangladesh Rural Advancement Committee (BRAC) has provided interventions in 250 upazilas in Bangladesh since 2006 with the aim of improving the health of the rural poor. The BRAC WASH program selects intervention areas on the basis of some criteria such as high poverty rate, poor sanitation coverage, and lack of access to safe water due to high arsenic, salinity, and other contaminants [7]. The program has adopted a holistic approach integrating water, sanitation, and hygiene components. The water component promotes use of safe water through a number of activities: (1) deep tubewell installation in arsenic-affected areas; (2) loan to construct tubewell platform in order to protect groundwater from pollutions; and (3) water quality testing [8]. Besides, awareness building and behavioral change remain at the core of the WASH program [9] to improve health and hygiene of the rural poor. The types of interventions vary according to households' economic status.

Earlier, we conducted a number of studies on waterand hygiene-related issues in intervention areas, such as use of tubewell water and water safety practices [8], women in water hygiene [10], and knowledge gap on hygiene and safe water [7]. Some impeding factors towards access to safe drinking are poverty, unhygienic sanitation practices, low groundwater levels, and impacts of natural hazards (e.g., arsenic, salinity, extreme weather events) [11]. The program assessed water safety in a crude way by some proxy indicators such as awareness on brick-built tubewell platform, its cleanliness, and no waterlogging at the bottom of the tubewell. To our knowledge, the present study on water quality assessment based on some water parameters has been the first study conducted for the BRAC WASH program. We aimed through this research to understand households' exposure to these water parameters according to their background characteristics which might have programmatic implications in the future. The present study measures drinking water quality with the application of weighted arithmetic WQI method based on some chemical parameters. These parameters used for drinking water quality assessment were selected as the requirement of BRAC WASH program. The relevance of the present study lies in programmatic implications by providing evidence-based and useful information on drinking water quality in a simple way. We expect that the findings will help in designing program interventions to ensure safe drinking water either by raising awareness about chemical contamination of water or by improving water quality through provision of hardware supply.

\section{Methods}

\section{Study design and area}

This study was part of our research on "The status of household WASH behaviors in rural Bangladesh," conducted in 24 randomly selected upazilas (5\% of total). The current study on the assessment of drinking water quality used a cross-sectional study design and was conducted in 12 out of 24 upazilas across the country: Alfadanga (Faridpur), Kendua (Netrokona), Shibchar (Madaripur), Rupsha (Khulna), Debhata (Satkhira), Patharghata (Barguna), Rangabali (Patuakhali), Anwara (Chittagong), Bijoynagar (Brahmanbaria), Shajahanpur (Bogra), Kamalganj (Moulvibazar), and Kurigram Sadar (Kurigram) (Fig. 1).

\section{Study procedure}

A total of 960 households from 24 upazilas (40 households in each upazila) were randomly selected for socioeconomic survey and arsenic test on the spot using test kit at household level. Twelve out of 24 upazilas were considered to collect water samples from drinking water sources and to test chemical parameters in the laboratory. A total of 542 water samples were collected from 293 randomly selected households. In each upazila, 20 out of 40 households were 


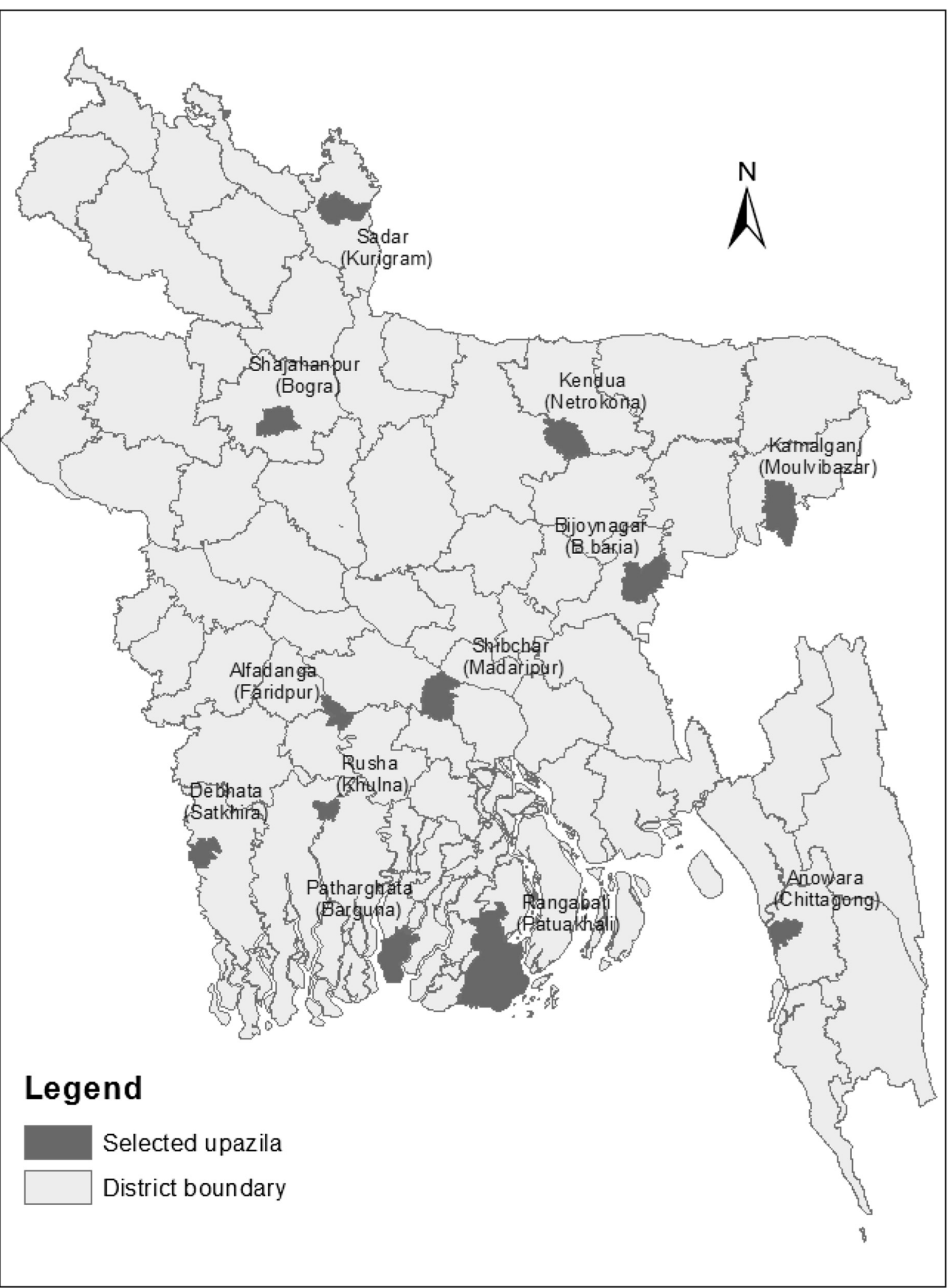

Fig. 1 Selected upazila for water sample collection 
initially selected for water samples collection. However, the total number of samples varied due to some reasons: (1) samples collected from shared tubewells increased the number of households; and (2) a few water samples were discarded due to label numbers being washed away, rendering them unidentifiable. Of the total water samples collected, same samples (293 samples) were used to test both iron and manganese levels in water. Similarly, the remaining 249 water samples were used to test both $\mathrm{pH}$ and salinity (Table 1).

A total of 36 research assistants were recruited and grouped into 12 to collect water samples from selected upazilas. They were trained intensively for 3 days and a field test was conducted nearby Dhaka prior to actual field survey. Groundwater samples from each tubewell were collected after $2 \mathrm{~min}$ of pumping in order to obtain deep water as the test sample. The water samples were collected in $100-\mathrm{mL}$ pre-washed bottles with watertight seals. The collected water samples were labeled with the household identification number and name of water parameters.

\section{Arsenic test on the spot}

A total of 960 households from 24 upazilas were visited for arsenic testing in the field. Simultaneously, a pre-tested structured questionnaire was used to obtain householdlevel information on socioeconomic condition. Of the total households visited, 66 and $31 \%$ households used shallow $(<300 \mathrm{ft})$ and deep $(\geq 300 \mathrm{ft})$ tubewells for collecting drinking water, respectively. Out of the total households using tubewells, 645 tubewells (424 shallow and 221 deep tubewells) were tested on the spot for arsenic using the "econo quick (EQ) arsenic test kit." The nature of EQ kit reading is quantitative. A color chart in a scale of values between 0.0 and $1.0 \mathrm{mg} / \mathrm{L}$ was used to record the arsenic status of water samples tested in the field. The EQ kit was preferred to use in field test because of its high accuracy (about $90 \%)$ of measuring arsenic status of the tubewells [12]. Drinking water sources of the remaining households (33\%) were not considered for arsenic testing for various reasons: tubewells of $29 \%$ of households had already been tested and declared arsenic free $(0.0 \mathrm{mg} / \mathrm{L})$ in the recent

Table 1 Sample distribution

\begin{tabular}{|c|c|c|c|c|c|c|}
\hline \multirow[b]{2}{*}{$\begin{array}{l}\text { Chemical } \\
\text { parameter }\end{array}$} & \multirow{2}{*}{$\begin{array}{l}\text { Spot test } \\
\text { Arsenic } \\
(\mathrm{As})\end{array}$} & \multicolumn{5}{|c|}{ Laboratory test } \\
\hline & & $\begin{array}{l}\text { Arsenic } \\
\text { (As) }\end{array}$ & $\begin{array}{l}\text { Manganese } \\
(\mathrm{Mn})\end{array}$ & $\begin{array}{l}\text { Iron } \\
(\mathrm{Fe})\end{array}$ & $\mathrm{pH}$ & $\begin{array}{l}\text { Salinity } \\
(\mathrm{NaCl})\end{array}$ \\
\hline $\begin{array}{l}\text { Households } \\
\text { visited }\end{array}$ & 960 & 293 & & & & \\
\hline Sample tested & 645 & 64 & 293 & 293 & 249 & 249 \\
\hline Total sample & 645 & 542 & & & & \\
\hline
\end{tabular}

past, and $4 \%$ used pond water for drinking and were excluded from arsenic testing.

\section{Testing of water samples using $\mathrm{pH}$ meter}

Acid-base balance is assessed by the $\mathrm{pH}$ value of water [13]. A controlled water $\mathrm{pH}$ is suggested in WHO guidelines to reduce adverse health consequences. According to the WHO guidelines of drinking water quality, exposure to both high and low $\mathrm{pH}$ values causes irritation the eyes, skin, and mucous membrane for humans [14]. Here, 123 water samples were randomly selected from the total samples collected to test the $\mathrm{pH}$. A portable $\mathrm{pH}$ meter (model PHS-25) was used in the BRAC head office to test the $\mathrm{pH}$. $\mathrm{pH}$ meter operating instructions were carefully followed: the meter was first calibrated by putting the electrode into standard buffer solutions of $\mathrm{pH} 6.86$ and $\mathrm{pH} 4.00$ at set temperature prior to being washed with distilled water and sample measurement.

\section{Methods used at laboratory for measuring parameters}

Arsenic results measured in the field using the testing kits were verified in the laboratory. About $10 \%$ of collected water samples were picked at random for laboratory validation. $\mathrm{pH}$ meter values were similarly crosschecked in the laboratory for validation. The other chemical contents (e.g., iron, manganese, and salinity) in water samples were also measured in the Water Quality Testing Laboratory of the NGO Forum for Public Health. The water samples were analyzed by flow-injection hydride generation atomic absorption spectrometry (FIHG-AAS) method for arsenic detection. The minimum detection level for this method was $3(\mu \mathrm{g} / \mathrm{L})$. Total arsenic was measured. The efficiency of field kits used by NGO Forum for arsenic testing was reported to have low failure rate (11 \% for Merck kit, $6.2 \%$ for NIPSOM), supporting high kit's performance in arsenic testing [15]. Manganese was analyzed in Flame (air-acetylene) AAS. The minimum detection limit of this method was $0.01 \mathrm{ppm}$. For both arsenic and manganese, AAS of Shimadzu (model: AA6300) was used at the laboratory. Iron was analyzed by phenanthroline method using UV-visible spectrophotometer where iron was brought into a solution, reduce to a ferrous state by treating with acid and hydroxylamine and 1, 10-phenanthroline. The minimum detection limit of this method was $0.05 \mathrm{ppm}$. UV-visible spectrophotometer of Shimadzu (model: UV-1601) was used at the laboratory. Salinity was measured at the laboratory by conductivity method using an appropriate electrode.

\section{Data analysis}

Descriptive statistics were used to analyze the mean, median, standard deviation, interquartile range (IQR), and frequency distribution of each parameter. The households' wealth index was developed based on ownership 
of valued items. Bangladesh and WHO guideline standards were considered in the evaluation of the number of household members exceeding acceptable drinking water limits. The exposure level of household members was analyzed by their background characteristics which included age, sex, education, economic status, and media access at home, NGO membership, wealth index, and type of water sources used. The households were classified as ultra-poor, poor, and non-poor as per the following criteria of the BRAC WASH program: households that owned less than $404.7 \mathrm{~m}^{2}$ of land, had no fixed source of income, or were headed by a female were classified as "ultra-poor"; households with land holdings between 404.7 and $4047 \mathrm{~m}^{2}$ and/or sold manual labor for a living were classified as "poor"; and households that did not fall into either of the above categories were classified as "non-poor." Wealth index was developed based on the ownership of valued items at household level.

\section{Weighted arithmetic Water Quality Index (WQI) method} The weighted arithmetic WQI method $[16,17]$ was applied to assess water suitability for drinking purposes. In this method, water quality rating scale, relative weight, and overall WQI were calculated by the following formulae:

$$
\boldsymbol{q}_{\boldsymbol{i}}=\left(\boldsymbol{C}_{\boldsymbol{i}} / \boldsymbol{S}_{\boldsymbol{i}}\right) \times 100
$$

where $q_{i}, C_{i}$, and $S_{i}$ indicated quality rating scale, concentration of $i$ parameter, and standard value of $i$ parameter, respectively.

Relative weight was calculated by

$$
w_{i}=1 / S_{i}
$$

where the standard value of the $i$ parameter is inversely proportional to the relative weight.

Finally, overall WQI was calculated according to the following expression:

$$
\mathrm{WQI}=\sum \boldsymbol{q}_{i} \boldsymbol{w}_{\boldsymbol{i}} / \sum \boldsymbol{w}_{\boldsymbol{i}}
$$

\section{Ethics statement}

The research protocol was approved by the ethical review committee of James P Grant School of Public Health, BRAC University.

\section{Results}

Demographic and socioeconomic profile of households

The background characteristics of households from whom water samples were collected for laboratory testing are shown in Table 2. A total of 293 households comprising 1491 members were included in the analysis. The proportions of male and female household members were 51 and $49 \%$, respectively. Over half of members had higher secondary education and above followed by secondary (22\%), primary (20\%), and no schooling (8\%). Members belonged to poor (37\%), ultra-poor (30 \%), and non-poor (32\%) economic groups. The study participants represented six divisions (highest administrative boundary of Bangladesh) including Dhaka and Khulna ( $8 \%$ in each), Chittagong and Barisal (about $28 \%$ in each), Rajshahi (11\%), and Sylhet (18\%). The majority had access to media (radio and/or television) at home (51\%), and $55 \%$ of the members had no NGO membership.

\section{$\mathrm{pH}$ levels in the drinking water}

The median of $\mathrm{pH}$ value was 7.4 , while IQR values at different sample sites varied between 0.2 and 0.4 , respectively. The highest frequency value was $\mathrm{pH} 7.4$ (34 \%) followed by $\mathrm{pH} 7.2$ (13\%) and $\mathrm{pH} 7.6$ (9\%). pH values at selected sites ranged between 6.6 and 8.4 (Table 3), within acceptable limits (6.5-8.5). The mean $\mathrm{pH}$ values in both shallow $(7.5 \pm 0.4)$ and deep tubewells $(7.4 \pm 0.3)$ varied, but median value was found the same in both types $(7.4 \mathrm{mg} / \mathrm{L})$.

\section{Manganese concentrations in drinking water}

In our samples, manganese concentrations varied between 0.1 and $5.5 \mathrm{mg} / \mathrm{L}$ with a median value of $0.2 \mathrm{mg} / \mathrm{L}$ (Table 3). At most sample sites, the median value exceeded the Bangladesh standard of $0.1 \mathrm{mg} / \mathrm{L}$, except Rangabali (Patuakhali) and Bijoynagar (B.Baria). The highest median value $(0.9 \mathrm{mg} / \mathrm{L})$ was observed in Shibchar (Madaripur), which exceeded the WHO standard of $0.4 \mathrm{mg} / \mathrm{L}$. Exposure to manganese in drinking water according to the household member characteristics is shown in Table 4. High exposure levels exceeding standards $(0.1 \mathrm{mg} / \mathrm{L})$ were found in Chittagong (27\%), Barisal (23 \%), and Sylhet (19\%). Those belonging to the lowest wealth group (26\%) had higher exposure to manganese $(>0.1 \mathrm{mg} / \mathrm{L})$ than those in the highest wealth group (16\%). When the WHO standard of $0.4 \mathrm{mg} / \mathrm{L}$ was considered, the majority of households (82\%) were within acceptable limits. According to Bangladesh standards, about half $(51 \%)$ of the households exceeded acceptable limits $(>0.1 \mathrm{mg} / \mathrm{L})$.

\section{Iron $(\mathrm{Fe})$ in drinking water}

The median iron concentration values in water exceeded WHO standards $(0.3 \mathrm{mg} / \mathrm{L})$ at all sample sites except Bijoynagar. The median iron values at a few sites exceeded Bangladesh limits $(1.0 \mathrm{mg} / \mathrm{L})$ (Table 3). The highest median iron concentration value was in Kamalganj (Moulvibazar) (2.3 $\mathrm{mg} / \mathrm{L})$ followed by Anwara (Chittagong) $(2.0 \mathrm{mg} / \mathrm{L})$, Shibchar (Madaripur) (2.0 mg/L), and Rupsha (Khulna) $(1.4 \mathrm{mg} / \mathrm{L})$. The lowest median value was observed in Bijoynagar (B.Baria) $(0.3 \mathrm{mg} / \mathrm{L})$. 
Table 2 Demographic and socioeconomic characteristics of households

\begin{tabular}{|c|c|c|}
\hline Characteristics & $\begin{array}{l}\text { Frequency } \\
\left(\mathrm{HH}^{\mathrm{a}} \text { members) }\right.\end{array}$ & Percentage (\%) \\
\hline \multicolumn{3}{|l|}{ Sex } \\
\hline Male & 757 & 50.8 \\
\hline Female & 734 & 49.2 \\
\hline \multicolumn{3}{|l|}{ Age(years) } \\
\hline$\leq 4$ & 106 & 7.2 \\
\hline $5-20$ & 516 & 34.9 \\
\hline $21-40$ & 455 & 30.8 \\
\hline $41-60$ & 300 & 20.3 \\
\hline$\geq 61$ & 102 & 6.9 \\
\hline \multicolumn{3}{|l|}{ Educational level } \\
\hline No schooling & 62 & 7.6 \\
\hline Primary & 166 & 20.3 \\
\hline Secondary & 177 & 21.6 \\
\hline Higher secondary and above & 414 & 50.5 \\
\hline \multicolumn{3}{|l|}{ Division } \\
\hline Dhaka & 115 & 7.7 \\
\hline Chittagong & 414 & 27.8 \\
\hline Rajshahi & 160 & 10.7 \\
\hline Khulna & 121 & 8.1 \\
\hline Barisal & 411 & 27.6 \\
\hline Sylhet & 270 & 18.1 \\
\hline \multicolumn{3}{|l|}{ Occupation } \\
\hline Agriculture & 123 & 9.0 \\
\hline Laborer (skilled/unskilled) & 123 & 9.0 \\
\hline Housewife/homestead task & 408 & 29.9 \\
\hline Service/professional & 87 & 6.4 \\
\hline Business & 88 & 6.5 \\
\hline Student & 427 & 31.3 \\
\hline Unemployed/disabled & 70 & 5.1 \\
\hline Others & 38 & 2.8 \\
\hline \multicolumn{3}{|l|}{ Household economic status } \\
\hline Ultra-poor & 452 & 30.3 \\
\hline Poor & 557 & 37.4 \\
\hline Non-poor & 482 & 32.3 \\
\hline \multicolumn{3}{|l|}{ Marital status } \\
\hline Unmarried & 692 & 46.4 \\
\hline Married & 728 & 48.8 \\
\hline Widow/separated/divorced & 71 & 4.8 \\
\hline \multicolumn{3}{|l|}{ Access to media at home } \\
\hline No access to media & 730 & 49.0 \\
\hline Access to media & 761 & 51.0 \\
\hline \multicolumn{3}{|l|}{ NGO membership } \\
\hline No membership & 816 & 54.7 \\
\hline
\end{tabular}

Table 2 Demographic and socioeconomic characteristics of households (Continued)

\begin{tabular}{lll}
\hline Member of any NGO & 675 & 45.3 \\
Wealth index & & \\
Lowest & 342 & 23.1 \\
Second & 301 & 20.3 \\
Middle & 265 & 17.9 \\
Fourth & 262 & 17.7 \\
Highest & 313 & 21.1 \\
Total & 1491 & 100 \\
${ }^{\text {aHousehold }}$ & &
\end{tabular}

About $7 \%$ of young children ( $\leq 4$ years) were exposed to iron levels in drinking water that exceeded WHO and Bangladesh standards. The highest exposure levels, exceeding the WHO's acceptable limit of $0.3 \mathrm{mg} / \mathrm{L}$, were in Barisal (29 \%) followed by Chittagong (23\%) and Sylhet (20 \%) (Table 4). In Dhaka, only $7 \%$ of household members were exposed to greater than $0.3 \mathrm{mg} / \mathrm{L}$ iron in drinking water. Only $18 \%$ households met WHO standards $(\leq 0.3 \mathrm{mg} / \mathrm{L})$, while a large proportion $(82 \%)$ were exposed to high concentrations of iron in drinking water $(>0.3 \mathrm{mg} / \mathrm{L})$. The median iron concentration in deep tubewells was slightly higher $(0.8 \mathrm{mg} / \mathrm{L})$ than in shallow tubewells $(0.7 \mathrm{mg} / \mathrm{L})$, although median values in both cases exceeded WHO and lower limit of Bangladesh standards.

\section{Salinity $(\mathrm{NaCl})$ levels}

Division-wise variations in sodium chloride levels in drinking water are shown in Table 4 . The highest proportion of household members exposed to more than $600 \mathrm{mg} / \mathrm{L}$ sodium chloride was found in Dhaka (40\%) followed by Barisal (35\%) and Khulna (26\%). Considering Bangladesh standards (upper limit $600 \mathrm{mg} / \mathrm{L}$ ), more females than males exceeded their exposure limits (54\% vs. $46 \%$ ) (Table 4). As shown in Table 3, excess sodium chloride was detected in Rupsha (Khulna) $(1050 \mathrm{mg} / \mathrm{L}$ ) when the upper limit of Bangladesh standard $(600 \mathrm{mg} / \mathrm{L})$ was considered.

\section{Arsenic (As) concentrations in drinking water}

Arsenic testing in the field revealed high arsenic concentrations exceeding Bangladesh standards in Shibchar (Madaripur), Biswanath (Sylhet), and Dhaka. A random sub-sample (over $10 \%$ ) was selected for laboratory validation, which showed that water samples collected from Shibchar (Madaripur) (median $0.05 \mathrm{mg} / \mathrm{L}$ ) and Alfadanga (Faridpur) (median $0.03 \mathrm{mg} / \mathrm{L}$ ) showed higher arsenic concentrations compared to other sample sites exceeding WHO standard (Table 3). About 68 and $77 \%$ 
Table 3 Regional variation in the values of chemical parameters of drinking water

\begin{tabular}{|c|c|c|c|c|c|c|}
\hline \multirow{2}{*}{ Sample site } & & \multicolumn{5}{|c|}{ Chemical parameter } \\
\hline & & $\mathrm{pH}$ & Manganese (Mn) (mg/L) & Iron (Fe) (mg/L) & Salinity (NaCl) (mg/L) & Arsenic (As) (mg/L) \\
\hline \multirow[t]{3}{*}{ Alfadanga (Faridpur) } & Median & 7.4 & 0.3 & 0.6 & 400 & 0.033 \\
\hline & IQR & 0.3 & 0.4 & 2.5 & 150 & 0.063 \\
\hline & Mean \pm SD & $7.4 \pm 0.4$ & $0.3 \pm 0.2$ & $2.0 \pm 2.9$ & $478.6 \pm 210.0$ & $0.047 \pm 0.034$ \\
\hline \multirow[t]{3}{*}{ Kendua (Netrokona) } & Median & 7.4 & 0.2 & 0.5 & 200 & 0.007 \\
\hline & IQR & 0.4 & 0.4 & 1.4 & 100 & 0.01 \\
\hline & Mean \pm SD & $7.6 \pm 0.4$ & $0.3 \pm 0.3$ & $1.1 \pm 1.7$ & $197.0 \pm 243.0$ & $0.031 \pm 0.053$ \\
\hline \multirow[t]{3}{*}{ Shibchar } & Median & 7.4 & 0.9 & 2 & 450 & 0.045 \\
\hline & IQR & 0.4 & 0.9 & 3.9 & 500 & 0.028 \\
\hline & Mean \pm SD & $7.4 \pm 0.3$ & $0.9 \pm 0.6$ & $2.0 \pm 2.8$ & $584.4 \pm 352.1$ & $0.057 \pm 0.037$ \\
\hline \multirow[t]{3}{*}{ Rupsha (Khulna) } & Median & 7.4 & 0.2 & 1.4 & 1050 & 0.008 \\
\hline & IQR & 0.3 & 0.1 & 2.4 & 1050 & 0.013 \\
\hline & Mean \pm SD & $7.4 \pm 0.2$ & $0.2 \pm 0.1$ & $2.3 \pm 2.0$ & $1180 \pm 723.0$ & $0.009 \pm 0.006$ \\
\hline \multirow[t]{3}{*}{ Debhata (Satkhira) } & Median & 7.4 & 0.2 & 0.8 & 400 & 0.003 \\
\hline & IQR & 0.2 & 0.2 & 0.5 & 150 & 0.001 \\
\hline & Mean \pm SD & $7.5 \pm 0.4$ & $0.3 \pm 0.3$ & $1.4 \pm 1.6$ & $391.7 \pm 79.3$ & $0.006 \pm 0.006$ \\
\hline \multirow[t]{3}{*}{ Patharghata (Barguna) } & Median & 7.4 & 0.2 & 0.6 & 100 & 0.004 \\
\hline & IQR & 0.4 & 0.2 & 0.4 & 400 & 0.000 \\
\hline & Mean \pm SD & $7.4 \pm 0.3$ & $0.2 \pm 0.1$ & $0.9 \pm 0.7$ & $717.5 \pm 1133.2$ & 0.004 \\
\hline \multirow[t]{3}{*}{ Rangabali (Patuakhali) } & Median & 7.4 & 0.1 & 0.4 & 500 & 0.005 \\
\hline & IQR & 0.2 & 0.1 & 0.2 & 100 & 0.002 \\
\hline & Mean \pm SD & $7.5 \pm 0.3$ & $0.1 \pm 0.1$ & $0.4 \pm 0.3$ & $557.5 \pm 84.4$ & $0.004 \pm 0.001$ \\
\hline \multirow[t]{3}{*}{ Anwara (Chittagong) } & Median & - & 0.2 & 2.0 & - & 0.006 \\
\hline & IQR & - & 1.5 & 3.2 & - & 0.008 \\
\hline & Mean \pm SD & - & $0.7 \pm 0.8$ & $2.8 \pm 3.2$ & - & $0.009 \pm 0.008$ \\
\hline \multirow[t]{3}{*}{ Bijoynagar (B.Baria) } & Median & 7.3 & 0.1 & 0.3 & 100 & - \\
\hline & IQR & 0.0 & 0.2 & 0.9 & 0 & - \\
\hline & Mean \pm SD & - & $0.3 \pm 0.4$ & $0.6 \pm 0.8$ & - & - \\
\hline \multirow[t]{3}{*}{ Shajahanpur (Bogra) } & Median & 7.4 & 0.3 & 0.7 & 100 & - \\
\hline & IQR & 0.5 & 0.4 & 0.9 & 0 & - \\
\hline & Mean \pm SD & $7.4 \pm 0.3$ & $0.4 \pm 0.4$ & $1.2 \pm 1.4$ & - & - \\
\hline \multirow[t]{3}{*}{ Kamlganj (Moulvibazar) } & Median & - & 0.3 & 2.3 & - & 0.075 \\
\hline & IQR & - & 0.5 & 6.2 & - & 0.000 \\
\hline & Mean \pm SD & - & $0.4 \pm 0.3$ & $5.0 \pm 6.2$ & - & - \\
\hline \multirow[t]{3}{*}{ Sadar (Kurigram) } & Median & 7.4 & - & - & 100 & - \\
\hline & IQR & 0.5 & - & - & 100 & - \\
\hline & Mean $\pm S D$ & $7.5 \pm 0.4$ & - & - & $135.0 \pm 62.2$ & - \\
\hline \multicolumn{2}{|l|}{ Bangladesh standard } & $6.5-8.5$ & 0.1 & $0.3-1.0$ & $150-600$ & 0.05 \\
\hline \multicolumn{2}{|l|}{ WHO standard } & $6.5-8.5$ & 0.4 & 0.3 & 250 & 0.01 \\
\hline
\end{tabular}

Water pH in shallow tubewell: median (7.4), IQR (0.5), mean (7.5), SD (0.38). Water pH in deep tubewell: median (7.4), IQR (0.4), mean (7.4), SD (0.32)

of household members in the Dhaka division were exposed to higher levels of arsenic with respect to WHO (0.01 mg/L) and Bangladesh standards $(0.05 \mathrm{mg} / \mathrm{L})$, respectively (Table 4).
Water Quality Index (WQI)

Drinking water was considered excellent in Kurigram Sadar and Rangabali (Patuakhali) (WQI value $<50$ ) (Table 5 ). Of the total sample sites, $33 \%$ (4 out of 12 sites) 
Table 4 Status of chemical parameters by WHO and Bangladesh drinking water standard (\%)

\begin{tabular}{|c|c|c|c|c|c|c|c|c|c|c|c|c|c|c|c|c|}
\hline \multirow[t]{3}{*}{ Characteristics } & \multicolumn{8}{|c|}{ WHO drinking water standard (mg/L) } & \multicolumn{8}{|c|}{ Bangladesh drinking water standard (mg/L) } \\
\hline & \multicolumn{2}{|l|}{$\mathrm{Mn}$} & \multicolumn{2}{|l|}{$\mathrm{Fe}$} & \multicolumn{2}{|l|}{$\mathrm{NaCl}$} & \multicolumn{2}{|l|}{ As } & \multicolumn{2}{|l|}{$\mathrm{Mn}$} & \multicolumn{2}{|l|}{$\mathrm{Fe}$} & \multicolumn{2}{|l|}{$\mathrm{NaCl}$} & \multicolumn{2}{|l|}{ As } \\
\hline & $\leq 0.4$ & $>0.4$ & $\leq 0.3$ & $>0.3$ & $\leq 250$ & $>250$ & $\leq 0.01$ & $>0.01$ & $\leq 0.1$ & $>0.1$ & $\leq 1.0$ & $>1.0$ & $\leq 600$ & $>600$ & $\leq 0.05$ & $>0.05$ \\
\hline \multicolumn{17}{|l|}{ Sex } \\
\hline Male & 50.3 & 52.8 & 50.8 & 50.8 & 51.7 & 50.3 & 50.0 & 52.3 & 50.3 & 51.2 & 51.8 & 48.9 & 51.9 & 46.1 & 50.2 & 56.7 \\
\hline Female & 49.7 & 47.2 & 49.2 & 49.2 & 48.3 & 49.7 & 50.0 & 47.7 & 49.7 & 48.8 & 48.2 & 51.1 & 48.1 & 53.9 & 49.8 & 43.3 \\
\hline$p$ value & 0.462 & & 0.995 & & 0.643 & & 0.691 & & 0.709 & & 0.290 & & 0.109 & & 0.495 & \\
\hline \multicolumn{17}{|l|}{ Age(years) } \\
\hline$\leq 4$ & 7.1 & 8.3 & 8.7 & 7.0 & 7.2 & 7.1 & 7.1 & 6.5 & 6.5 & 7.8 & 4.6 & 6.9 & 7.2 & 7.0 & 7.0 & 6.7 \\
\hline $5-20$ & 35.6 & 30.9 & 37.0 & 34.3 & 35.0 & 35.7 & 35.6 & 39.3 & 37.4 & 32.5 & 34.5 & 34.5 & 35.5 & 34.9 & 35.1 & 53.3 \\
\hline $21-40$ & 30.4 & 32.8 & 28.7 & 31.3 & 32.0 & 32.1 & 31.8 & 23.4 & 29.2 & 32.3 & 30.3 & 32.2 & 31.9 & 32.8 & 30.1 & 20.0 \\
\hline $41-60$ & 20.0 & 21.5 & 18.9 & 20.5 & 20.2 & 17.9 & 17.2 & 24.3 & 20.4 & 20.2 & 20.9 & 18.9 & 19.0 & 17.9 & 19.3 & 20.0 \\
\hline$\geq 61$ & 7.0 & 6.4 & 6.8 & 6.9 & 5.6 & 7.2 & 8.4 & 6.5 & 6.5 & 7.2 & 6.7 & 7.5 & 6.4 & 7.4 & 8.5 & 90.0 \\
\hline$p$ value & \multicolumn{2}{|c|}{0.6623} & \multicolumn{2}{|l|}{0.720} & \multicolumn{2}{|l|}{0.707} & \multicolumn{2}{|l|}{0.358} & \multicolumn{2}{|l|}{0.318} & 0.805 & & 0.975 & & 0.199 & \\
\hline Educational level & & & & & & & & & & & & & & & & \\
\hline No education & 7.1 & 9.8 & 8.8 & 7.3 & 9.5 & 6.8 & 7.5 & 5.0 & 5.7 & 9.4 & 8.0 & 7.1 & 8.0 & 7.1 & 6.9 & 5.0 \\
\hline Primary & 20.0 & 21.2 & 19.2 & 20.4 & 25.9 & 22.2 & 15.0 & 28.3 & 19.9 & 20.7 & 20.9 & 18.9 & 26.3 & 12.7 & 17.9 & 30.0 \\
\hline Secondary & 20.6 & 26.5 & 14.4 & 22.8 & 17.2 & 21.2 & 21.1 & 20.0 & 19.4 & 23.8 & 19.5 & 24.2 & 19.2 & 21.4 & 20.8 & 20.0 \\
\hline Higher secondary and above & 52.2 & 42.4 & 57.6 & 49.4 & 47.5 & 49.8 & 56.4 & 46.7 & 55.1 & 46.2 & 51.7 & 49.8 & 46.5 & 58.7 & 54.3 & 45.0 \\
\hline$p$ value & 0.166 & & 0.152 & & 0.257 & & 0.175 & & $0.032^{*}$ & & 0.443 & & $0.010^{* *}$ & & 0.623 & \\
\hline Household economic status & & & & & & & & & & & & & & & & \\
\hline Ultra-poor & 30.1 & 32.2 & 24.4 & 31.8 & 21.3 & 19.1 & 26.4 & 27.5 & 27.7 & 32.8 & 30.2 & 30.4 & 20.3 & 18.5 & 25.9 & 36.7 \\
\hline Poor & 36.1 & 42.7 & 36.1 & 37.5 & 42.3 & 38.6 & 33.5 & 42.2 & 39.0 & 35.8 & 36.0 & 38.3 & 41.9 & 31.9 & 33.6 & 63.3 \\
\hline Non-poor & 33.8 & 25.1 & 39.5 & 30.7 & 36.4 & 42.3 & 40.1 & 30.3 & 33.3 & 31.4 & 33.8 & 31.3 & 37.7 & 49.6 & 40.5 & 0.0 \\
\hline$p$ value & $0.018^{*}$ & & $0.010^{*}$ & & 0.107 & & 0.168 & & $0.097^{*}$ & & 0.551 & & $0.003^{* * *}$ & & $0.000^{* * *}$ & \\
\hline Access to media at home & & & & & & & & & & & & & & & & \\
\hline No access to media & 48.4 & 50.9 & 35.7 & 51.7 & 82.6 & 58.3 & 65.3 & 35.8 & 46.6 & 51.2 & 52.7 & 40.8 & 66.2 & 76.7 & 56.1 & 56.7 \\
\hline Access to media & 51.6 & 49.1 & 64.3 & 48.3 & 17.4 & 41.7 & 34.7 & 64.2 & 53.4 & 48.8 & 47.3 & 59.2 & 33.8 & 23.3 & 43.9 & 43.3 \\
\hline$p$ value & 0.447 & & $0.000^{*}$ & & $0.000^{* *}$ & & $0.000^{* * *}$ & & $0.070^{*}$ & & $0.000^{*}$ & & $0.002^{* * *}$ & & 0.950 & \\
\hline NGO membership & & & & & & & & & & & & & & & & \\
\hline No membership & 54.0 & 57.3 & 56.4 & 54.2 & 60.7 & 59.3 & 58.7 & 61.5 & 54.0 & 55.4 & 52.2 & 59.2 & 58.6 & 65.5 & 60.4 & 50.0 \\
\hline Member of any NGO & 46.0 & 42.7 & 43.6 & 45.8 & 39.3 & 40.7 & 41.3 & 38.5 & 46.0 & 44.6 & 47.8 & 40.8 & 41.4 & 34.5 & 39.6 & 50.0 \\
\hline$p$ value & 0.324 & & 0.513 & & 0.622 & & 0.622 & & 0.579 & & $0.009^{*}$ & & $0.051^{* *}$ & & 0.265 & \\
\hline Wealth index & & & & & & & & & & & & & & & & \\
\hline Lowest & 22.1 & 27.0 & 28.9 & 21.7 & 22.6 & 21.1 & 38.4 & 0.0 & 20.1 & 25.9 & 25.0 & 20.9 & 22.0 & 20.4 & 28.8 & 0.0 \\
\hline Second & 20.2 & 21.7 & 17.3 & 21.2 & 18.3 & 19.1 & 12.2 & 9.2 & 15.6 & 24.8 & 21.5 & 18.2 & 17.2 & 25.8 & 11.1 & 13.3 \\
\hline Middle & 19.0 & 12.4 & 19.2 & 17.5 & 31.2 & 24.3 & 28.3 & 20.2 & 21.2 & 14.7 & 20.9 & 13.2 & 26.6 & 29.4 & 26.9 & 13.3 \\
\hline Fourth & 17.0 & 20.6 & 16.9 & 17.8 & 20.2 & 17.2 & 5.5 & 48.6 & 17.1 & 18.2 & 16.4 & 19.8 & 20.0 & 11.3 & 15.2 & 60.0 \\
\hline Highest & 21.6 & 18.4 & 17.7 & 21.8 & 7.7 & 18.3 & 15.6 & 22.0 & 26.0 & 16.4 & 16.2 & 28.0 & 14.2 & 13.1 & 18.0 & 13.3 \\
\hline$p$ value & $0.031^{*}$ & & $0.066^{*}$ & & $0.000^{* *}$ & & $0.000^{* * *}$ & & $0.000^{*}$ & & $0.000^{*}$ & & $0.004^{* * *}$ & & $0.000^{* * *}$ & \\
\hline Water sources by type & & & & & & & & & & & & & & & & \\
\hline Shallow tubewell & 38.2 & 78.3 & 72.9 & 39.4 & 74.2 & 28.4 & 31.0 & 70.6 & 43.0 & 47.8 & 42.9 & 47.2 & 52.8 & 21.1 & 40.2 & 76.7 \\
\hline Deep tubewell & 50.0 & 21.7 & 27.1 & 48.8 & 1.8 & 68.6 & 69.0 & 29.4 & 49.4 & 40.4 & 42.2 & 50.9 & 33.1 & 78.9 & 59.8 & 23.3 \\
\hline Others & 11.8 & 0.0 & 0.0 & 11.8 & 24.1 & 2.9 & 0.0 & 0.0 & 7.6 & 11.8 & 14.9 & 1.9 & 14.1 & 0.0 & 0.0 & 0.0 \\
\hline$p$ value & $0.000^{*}$ & & $0.000^{*}$ & & $0.000^{* *}$ & & $0.000^{* * *}$ & & $0.000^{*}$ & & $0.000^{*}$ & & $0.000^{* * *}$ & & $0.000^{* * *}$ & \\
\hline
\end{tabular}


Table 4 Status of chemical parameters by WHO and Bangladesh drinking water standard (\%) (Continued)

\begin{tabular}{lllllllllllllllll}
\hline Division & 6.6 & 12.7 & 11.7 & 6.8 & 36.0 & 40.1 & 9.1 & 67.9 & 6.5 & 8.9 & 7.4 & 8.5 & 38.1 & 39.7 & 22.7 & 76.7 \\
Dhaka & 25.4 & 39.7 & 50.8 & 23.0 & 1.6 & 0.0 & 16.5 & 9.2 & 28.7 & 26.9 & 27.2 & 28.4 & 0.8 & 0.0 & 15.6 & 0.0 \\
Chittagong & 9.3 & 17.2 & 10.2 & 10.8 & 2.7 & 0.0 & - & - & 8.3 & 13.1 & 10.8 & 9.0 & 1.4 & 0.0 & - & - \\
Rajshahi & 9.5 & 1.5 & 0.0 & 9.8 & 1.0 & 19.8 & 20.2 & 16.5 & 6.5 & 9.7 & 7.1 & 9.5 & 9.0 & 25.9 & 20.9 & 0.0 \\
Khulna & 32.3 & 5.2 & 20.3 & 29.0 & 24.1 & 38.5 & 54.1 & 0.0 & 32.9 & 22.5 & 41.2 & 6.9 & 32.2 & 34.5 & 40.8 & 0.0 \\
Barisal & 16.9 & 23.6 & 7.1 & 20.4 & 34.6 & 1.7 & 0.0 & 6.4 & 17.2 & 19.0 & 6.3 & 37.6 & 18.5 & 0.0 & 0.0 & 23.3 \\
Sylhet & $0.000^{* * *}$ & $0.000^{* * *}$ & $0.000^{* * *}$ & & $0.000^{* * *}$ & & $0.000^{* * *}$ & $0.000^{* * *}$ & $0.000^{* * *}$ & $0.000^{* * *}$ \\
$\begin{array}{l}P \text { value } \\
\mathrm{HH}\end{array}$ & 82 & 18 & 17.8 & 82.2 & 40.6 & 59.4 & 68.9 & 31.1 & 48.7 & 51.3 & 61.4 & 38.6 & 81.6 & 18.4 & 91.5 & 8.5 \\
$\mathrm{HH}(\%)$ & 82 & 18 & 18 & 82 & 41 & 59 & 69 & 31 & 49 & 51 & 61 & 39 & 82 & 18 & 91.5 & 8.5 \\
\hline
\end{tabular}

${ }^{*} p<0.10 ;{ }^{* *} p<0.05 ;{ }^{* * *} p<0.01$

aHousehold

had good-quality drinking water (WQI value $<100$ ) and the majority $(67 \%)$ had poor-quality drinking water (WQI value $>100$ ). Quality of drinking water was found very poor in Anwara (Chittagong) and Kamalganj (Moulvivazar), while water was categorized as unsuitable for drinking only in Shibchar (Madaripur).

\section{Discussion}

Assessment of drinking water quality is a timely requirement amid emerging public health problems in this context where availability of safe water is at risk due to natural and man-made activities. This cross-sectional study conducted across the country aimed at measuring drinking water quality using WQI which delivered messages on the composite effect of chemical parameters on water. The present study is a fact finding or exploratory

Table 5 Computed water quality values for sample sites

\begin{tabular}{|c|c|c|}
\hline \multirow[t]{2}{*}{$\begin{array}{l}\text { Sample site upazila } \\
\text { name (district name) }\end{array}$} & \multirow[t]{2}{*}{ WQI value } & $\begin{array}{l}\text { Water quality classification based } \\
\text { on computed WQI values in sample } \\
\text { sites }\end{array}$ \\
\hline & & $\begin{array}{l}<50=\text { excellent; } 50-100=\text { good } \\
\text { water; } 101-200=\text { poor water; } \\
201-300=\text { very poor water, } \\
>300=\text { water unsuitable for drinking }\end{array}$ \\
\hline Rangabali (Patuakhali) & 40.05 & Excellent water \\
\hline Sadar (Kurigram) & 11.79 & Excellent water \\
\hline Rupsha (Khulna) & 92.14 & Good water \\
\hline Patharghata (Barguna) & 75.35 & Good water \\
\hline Alfadanga (Faridpur) & 169.44 & Poor water \\
\hline Kendua (Netrokona) & 142.51 & Poor water \\
\hline Debhata (Satkhira) & 113.18 & Poor water \\
\hline Shajahanpur (Bogra) & 135.67 & Poor water \\
\hline Bijoynagar (B.baria) & 111.83 & Poor water \\
\hline Anwara (Chittagong) & 253.29 & Very poor water \\
\hline Kamalganj (Moulvibazar) & 258.36 & Very poor water \\
\hline Shibchar (Madaripur) & 371.50 & Water unsuitable for drinking \\
\hline
\end{tabular}

study contributing to designing and improving program interventions which cover a larger population including high arsenic, high saline prone coastal areas. There is duality about spatial and temporal variations of some chemical parameters. A periodic assessment on arsenic concentration depicts no association with seasonal variations, while repeated assessment of arsenic contents in water based on seasons is assumed to bring little value in health surveillance [18]. In contrast, seasonal and spatial variations of arsenic concentrations in groundwater have been reported by Shrestha et al. [19].

The study findings revealed that drinking water was slightly alkaline, although the ideal $\mathrm{pH}$ for human consumption is stated to be 7.4 [20]. A controlled $\mathrm{pH}$ of water is suggested in $\mathrm{WHO}$ guideline to reduce the corrosion and contamination of drinking water having health consequences. Water $\mathrm{pH}$ is influenced by a number of factors including rock and soil composition and the presence of organic materials or other chemicals. Napacho and Manyele [21] found that $\mathrm{pH}$ values in shallow tubewells varied between 6.7 and 8.3 due to dissolved minerals from the soil and rocks. They further explained higher alkalinity by the presence of two common minerals, calcium and magnesium, affecting the hardness of the water. On the other hand, water with low $\mathrm{pH}$ values is meant to be acidic, soft, and corrosive.

The median value of manganese concentrations exceeded Bangladesh standard at most of the study sites. Other Bengali studies have reported higher manganese levels in drinking water in terms of WHO standards [22]. For example, Islam et al. [23] reported that $52 \%$ of pond-sand filter and $45 \%$ of pond water exceeded Bangladesh drinking water standards. The median value at our sample sites was relatively lower than some previous findings (about 0.8 and $0.9 \mathrm{mg} / \mathrm{L}$ ) $[24,25]$ but higher than the $0.1 \mathrm{mg} / \mathrm{L}$ reported by Bouchard [26].

Children are reported to be particularly vulnerable to higher manganese concentrations due to their low 
protective mechanisms. Approximately $8 \%$ of children were exposed to excess manganese concentrations that exceeded both WHO and Bangladesh standards ( $>0.4$ and $>0.1 \mathrm{mg} / \mathrm{L}$, respectively). We found higher exposure to manganese in lowest wealth group. This finding has similarity with the other study conducted in Araihazar, Bangladesh [27]. Less exposure among the infants was reported by mothers who had access to TV. Besides, participants living in poor-quality housing type (mud vs. concrete) were more likely to report exposure among the infants. Several studies have reported that exposure to high manganese concentrations threatens children's cognitive [28], behavioral, and neuropsychological health [25]. However, the potential impact of lower exposure and interactions with other metals are less well characterized. Infants and children are reported to be more susceptible to manganese toxicity than adults [27], and a number of Bangladesh studies have shown that children's intellectual function, and consequently their academic achievement, was adversely affected by manganese exposure in drinking water [22, 25, 27]. Contradictory to these findings, a higher manganese level in drinking water was shown to be protective against fetal loss during pregnancy of undernourished women in Bangladesh [29].

In most of the sample sites (9 out of 12 sites), iron content in drinking water exceeded upper acceptable limit $(1.0 \mathrm{mg} / \mathrm{L})$ of Bangladesh standard. A previous study in rural Bangladesh revealed 50 times higher iron concentrations (mean value $16.7 \mathrm{mg} / \mathrm{L}$ ) in ground water than WHO's limit $(0.3 \mathrm{mg} / \mathrm{L})$ and reported that $47 \%$ of women consumed above the daily limit of iron $(45 \mathrm{mg})$, likely to increase the risk of health problems [30]. Consumption of $>30 \mathrm{mg}$ of iron per day in drinking water was associated with a reduced risk of anemia in individuals without thalassemia [31]. In Gaibandha, half of female respondents consuming $>42 \mathrm{mg}$ of iron from drinking water stayed within tolerable limits. If this limit were exceeded, however, the populations would be likely to experience health-related problems including gastrointestinal distress, zinc absorption, and others [32].

Approximately $2 \%$ of women in developed countries but $50 \%$ in developing countries are anemic, contributing to high rates of maternal mortality in developing countries [33]. Iron-deficiency anemia is one of the top ten contributing factors to the global burden of diseases and is considered a public health problem with a high risk of morbidity and mortality in pregnant women and young children [34]. In our study, about half of the female participants were exposed to higher iron concentrations in drinking water which exceeded both WHO and Bangladesh standard. The health impacts of exceeding recommended WHO levels of chemical substances such as iron are often not well documented [32]. There is a duality to iron concentrations: on the one hand, iron deficiency can cause anemia and fatigue, while on the other, excess iron can cause multiple organ dysfunction (e.g., liver fibrosis and diabetes) [35]. In a 10-year period of study in Bangladesh, the prevalence of anemia in women of reproductive age ranged between 23 and $95 \%$ depending on age, pregnancy status, and residency. However, more recent studies have reported iron deficiency as the most important determinant of 7 to $60 \%$ of anemia cases in Bangladesh [36].

Salinity in drinking water was found higher $(>600 \mathrm{mg} / \mathrm{L})$ only in Rupsha (Khulna) and Patharghata (Barguna). Geographically, these two upazilas are coastal areas. Salinity problems in coastal regions are assumed to be the effects of climate change [37], although industrial and domestic wastes [38] and geological and soil characteristics [21] are also thought to contribute. Bangladesh is at the forefront of the negative effects of climate change and has faced dramatic rises in sea level over the last three decades. Approximately 20 million people living in coastal Bangladesh [24] are dependent on tubewells, rivers, and ponds for drinking water, and these sources are increasingly becoming saline due to rising sea levels [39]. Salinity has intruded over $100 \mathrm{~km}$ inland from the Bay of Bengal with consequent health impacts: in a 2008 survey, higher rates of preeclampsia and hypertension were reported in the coastal than non-coastal population [40]. Consistent with this, Khan et al. [41] reported that hypertensive disorders were associated with salinity in drinking water. Furthermore, reducing salt consumption from the global estimated levels of 9-12 g/day [42] to an acceptable limit of $5 \mathrm{~g} /$ day [43] would be predicted to reduce blood pressure and stroke/cardiovascular disease by 23 and $17 \%$, respectively [44].

Most households in Dohar, Shibchar, and Sonargaon used shallow tubewells for drinking, which were affected by high levels of arsenic. In Shibchar (West Kakor village), most tubewells were affected by arsenic, and the villagers were unaware of which tubewell was arsenic free; therefore, they collected drinking water from any tubewell. In some cases (e.g., Sonargaon), people used arsenic-affected drinking water sources even though they knew that the water was contaminated and damaging to health. Bladder cancer risk is increased 2.7 and 4.2 times by arsenic exposure of 10 and $50 \mu \mathrm{g} / \mathrm{L}$ in water, respectively. In this study, there was an $83 \%$ chance of developing bladder cancer and a $74 \%$ probability of mortality at a $50 \mu \mathrm{g} / \mathrm{L}$ exposure level. Mortality rates are $30 \%$ higher at 150 than $10 \mu \mathrm{g} / \mathrm{L}$ [45]. According to a national survey conducted in 2009 by UNICEF/BBS (2011), 53 and 22 million people were exposed to arsenic according to WHO and BDWS standards, respectively. Arsenic has been detected in the groundwater of 322 upazilas (subdistricts) and 61 districts in Bangladesh [46]. The health 
effects of prolonged and excessive inorganic arsenic exposure include arsenicosis, skin diseases, skin cancers, internal cancers (bladder, kidney, and lung), diabetes, raised blood pressure, and reproductive disorders [47].

The overall suitability of drinking water was assessed using a combined measure of water quality parameters: the WQI. The chemical parameters $(\mathrm{pH}$, iron, manganese, salinity, and arsenic) of water samples were used to calculate the WQI value at each site. We applied the weighted arithmetic WQI method to calculate WQI values. In this method, the permissible WQI value for drinking is considered to be 100 , the water quality being considered poor if the value exceeded this acceptable limit. Water quality was found excellent only in Rangabali (Patuakhali) and Kurigram Sadar. The water was considered excellent at these sites mainly due to low chemical parameter values contributing to lower composite effect on drinking water quality. In Shibchar (Madaripur), water was categorized as unsuitable for drinking, mainly due to high manganese and arsenic levels found in water at these sites. At most sample sites (e.g., Alfadanga, Kendua, Debhata, Shajahanpur, and Bijoynagar), water was classified as "poor" for drinking due to high manganese values. Moreover, arsenic was also found to be high in Alfadanga (Faridpur) and Kendua (Netrokona). However, in Anwara (Chittagong) and Kamalganj (Moulvibazar), the chemical parameter values in the water samples were very high and contributed to very poor-quality drinking water.

Most respondents at the sample sites used shallow tubewells to obtain drinking water due to lower installation costs. In some areas, such water from shallow tubewells was reported to have high iron and arsenic levels. In coastal districts such as Barguna, Satkhira, and Khulna, water from both shallow and deep tubewells were salty, as reported by the respondents. Yisa and Jimoh [16] reported higher levels of iron and manganese that contributed to poor-quality drinking water. These characteristics are consistent with unplanned waste disposal, agricultural run-off including pesticide or fertilizer, and other environmentally hazardous activities polluting surface water [48].

The study had some limitations. This study embraced cross-sectional study design. However, it would have been better to collect samples throughout the year addressing seasonality and depth of wells. We could not collect data on other WHO-recommended parameters which was beyond our scope of work. Therefore, the analysis has been limited to few water parameters as the requirement of BRAC WASH program and due to resource constraints. Measuring other WHO-recommended chemical parameters might have been a future concern for the program. In addition, water $\mathrm{pH}$ would have been tested on the spot using $\mathrm{pH}$ meter which was not possible for this study due to limited resources. The limitations observed in this study highlight the insights of future scope of work for research divisions and WASH program.

\section{Conclusions}

Here, we report that drinking water in Bangladesh was mainly alkaline with $\mathrm{pH}$ values within acceptable limits. According to $\mathrm{WHO}$ standards, a greater proportion of household members are exposed to excessive amounts of iron compared to manganese ( $82 \%$ vs. $18 \%$ ). About half of households exceeded acceptable limits of manganese exposure when considering Bangladeshi standards. Majority of the households used poor quality of drinking water according to WQI values. Higher values of iron, manganese, and arsenic reduced drinking water quality. Awareness raising on chemical contents in drinking water at household level is required to improve public health.

\begin{abstract}
Abbreviations
As: arsenic; BRAC: Bangladesh Rural Advancement Committee; CDC: Centers for Disease Control; EQ: econo quick; Fe: iron; IQR: interquartile range; MDG: Millennium Development Goals; Mn: manganese; $\mathrm{NaCl}$ : sodium chloride; NGO: non-government organization; UNICEF: United Nations International Children's Emergency Fund; WASH: Water, Sanitation and Hygiene; WHO: World Health Organization; WQI: Water Quality Index.
\end{abstract}

\section{Competing interests}

The authors declare that they have no competing interests.

\section{Authors' contributions}

TA, MR, FTJ, and FA conceived and designed the study. SKM, TRC, and DD contributed to the data collection. TA, FTJ, and MR conducted the data analysis and participated in the result interpretation. TA and FTJ wrote the manuscript. $\mathrm{MR}, \mathrm{MAl}$, and MKB commented on the manuscript draft. All authors read and approved the final manuscript.

\section{Acknowledgements}

We would like to thank the respondents who provided valuable information and time for this study. We thank all the interviewers involved in the data collection and the field staff of the BRAC WASH program for their assistance in conducting this study. We acknowledge the Data Management Unit of RED for their support in data entry and cleaning. Finally, we acknowledge the Government of the Netherlands for funding the study. We thank the Nextgenediting Global Initiative (www.nextgenediting.com) for editorial assistance.

\section{Author details}

1BRAC Research and Evaluation Division, BRAC Centre, 75 Mohakhali, Dhaka 1212, Bangladesh. ${ }^{2}$ BRAC Tuberculosis Programme, BRAC Centre, 75 Mohakhali, Dhaka 1212, Bangladesh. ${ }^{3}$ BRAC Water, Sanitation and Hygiene Programme, BRAC Centre, 75 Mohakhali, Dhaka 1212, Bangladesh.

Received: 18 September 2015 Accepted: 3 February 2016 Published online: 09 February 2016

\footnotetext{
References

1. Bureau of Indian Standards (BIS). Specification for drinking water. New Delhi, India: Food and Agricultural Division Council; 2012.

2. Central Pollution Control Board (CPCB). Guide manual: water and waste water. New Delhi, India: Central Pollution Control Board; 2013. Available: http:// www.cpcb.nic.in/upload/Latest/Latest_67_guidemanualw\&wwanalysis.pdf.

3. World Health Organization (WHO). Guideline for drinking water quality. 2012.

4. Ochuko U, Thaddeus O, Oghenero OA, John EE. A comparative assessment of water quality index (WQI) and suitability of river Ase for domestic water supply in urban and rural communities in Southern Nigeria. Int J Human Soc Sci. 2014;4(1):234-45.
} 
5. Tyagi S, Sharma B, Singh P, Dobhal R. Water quality assessment in terms of Water Quality Index. Am J Water Resour. 2013;1(3):34-8.

6. Chowdhury RM, Muntasir SY, Hossain MM. Water Quality Index of water bodies along Faridpur-Barisal Road in Bangladesh. Glob Eng Tech Rev. 2012;2:1-8.

7. Akter T, Ali ARMM. Factors influencing knowledge and practice of hygiene in Water, Sanitation and Hygiene (WASH) programme areas of Bangladesh Rural Advancement Committee. Rural Remote Health. 2014;14:2628. Online.

8. Dey NC, Rabbi SE. Studies on the impact of BRAC WASH-1 interventions: an overview. In: achievements of BRAC Water, Sanitation and Hygiene programme towards Millennium Development Goals and beyond. Dhaka: BRAC; 2013.

9. Rabbi SE, Dey NC. Exploring the gap between hand washing knowledge and practices in Bangladesh: a cross-sectional comparative study. BMC Public Health. 2013;13:89.

10. Dey NC, Akter T. Women in water-hygiene and sanitation management at households in rural Bangladesh: changes from baseline to end line survey. In: achievements of BRAC Water, Sanitation and Hygiene programme towards Millennium Development Goals and beyond. Dhaka: BRAC; 2013.

11. UNICEF. First annual high level meeting for sanitation and water for all aims to be a watershed for reaching the MDG targets. 2010. Available: http://www.unicef.org/bangladesh/media_6193.htm

12. George CM, Zheng Y, Graziano JH, Rasul SB, Hossain Z, Mey JL, et al. Evaluation of an arsenic test kit for rapid well screening in Bangladesh. Environ Sci Technol. 2012;46(20):11213-9.

13. World Health Organization (WHO). pH in drinking water. Guidelines for drinking water quality. Geneva: World Health Organization; 1996. Available: http://www.who.int/water_sanitation_health/dwq/chemicals/en/ph.pdf.

14. Ambica A. Groundwater quality characteristics study by using water quality index in Tambaram area, Chennai, Tamil Nadu. Middle East J Sci Res. 2014;20(11):1396-401.

15. Rahman MM, Mukherjee D, Sengupta MK, Chowdhury UK, Lodh D, Ranjan C, et al. Effectiveness and reliability of arsenic field testing kits: are the million dollar screening projects effective or not. Environ Sci Technol. 2002;36(24): 5385-94.

16. Yisa J, Jimoh T. Analytical studies on water quality index of river Landzu. Am J Appl Sci. 2010;7:453-8.

17. Tyagi S, Singh P, Sharma B, Singh R. Assessment of water quality for drinking purpose in District Pauri of Uttarkhand India. Appl Ecol Environ Sci. 2014:2(4):94-9.

18. Thundiyil JG, Yuan $Y$, Smith AH, Steinmaus $C$. Seasonal variation of arsenic concentration in wells in Nevada. Environ Res. 2007;104(3):367-73.

19. Shrestha SM, Rijal K, Pokhrel MR. Spatial distribution and seasonal variation of arsenic in groundwater of the Kathmandu Valley Nepal. J Inst Sci Technol. 2014;19(2):7-13.

20. Parker KT. What are the benefits of drinking alkaline water? 2013. Available: http://www.livestrong.com/article/498701-what-are-the-benefits-of-drinkingalkaline-water/.

21. Napacho ZA, Manyele SV. Quality assessment of drinking water in Temeke district (part II): characterization of chemical parameters. Afr J Environ Sci Technol. 2010:4:775-89.

22. Khan K, Wasserman GA, Liu X, Ahmed E, Parvez F, et al. Manganese exposure from drinking water and children's academic achievement. Neurotoxicology. 2012;33:91-7.

23. Islam MA, Karim MR, Higuchi T, Sakakibara H, Sekine M. Comparison of the trace metal concentration of drinking water supply options in southwest coastal areas of Bangladesh. Appl Water Sci. 2014;4:183-91.

24. Khan AE, Ireson A, Kovats S, Mojumder SK, Khusru A, et al. Drinking water salinity and maternal health in coastal Bangladesh: implications of climate change. Environ Health Perspect. 2011;119:1328-32.

25. Wasserman GA, Liu X, Parvez F, Ahsan H, Levy D, et al. Water manganese exposure and children's intellectual function in Araihazar Bangladesh. Environ Health Perspect. 2006;114:124-9.

26. Bouchard MF, Sauve S, Barbeau B, Legrand M, Brodeur M-E, et al. Intellectual impairment in school-age children exposed to manganese from drinking water. Environ Health Perspect. 2011;119:138-43.

27. Hafeman D, Factor-Litvak P, Cheng Z, van Geen A, Ahsan H. Association between manganese exposure through drinking water and infant mortality in Bangladesh. Environ Health Perspect. 2007;115:1107-12.

28. Roels HA, Bowler RM, Kim Y, Henn BC, Mergler D, et al. Manganese exposure and cognitive deficits: a growing concern for manganese neurotoxicity. Neurotoxicology. 2012;33:1-19.
29. Rahman SM, Åkesson A, Kippler M, Grandér M, Hamadani JD, et al. Elevated manganese concentrations in drinking water may be beneficial for fetal survival. PLoS ONE. 2013:8:e74119.

30. Merrill RD. Dietary iron may flow from tubewells in rural Bangladesh, 2012 Available: http://water.jhu.edu/magazine/dietary-iron-may-flow-from-tubewellsin-rural-bangladesh/.

31. Merrill RD, Shamim AA, Ali H, Labrique AB, Schulz K, et al. High prevalence of anemia with lack of iron deficiency among women in rural Bangladesh: a role for thalassemia and iron in groundwater. Asia Pac J Clin Nutr. 2012;21:416-24.

32. Merrill RD, Shamim AA, Ali H, Jahan N, Labrique AB, et al. Iron status of women is associated with the iron concentration of potable groundwater in rural Bangladesh. J Nutr. 2011;141:944-9.

33. Baby A, Venugopal J, D'silva R, Chacko S, Vineesha P, et al. Knowledge on management of anemia during pregnancy: a descriptive study. Med Health Sci. 2014:2:140-4.

34. Kraft $\mathrm{S}$. What is nutritional deficiency anemia? What causes nutritional deficiency anemia? 2014.

35. Heming $\mathrm{N}$, Montravers $\mathrm{P}$, Lasocki S. Iron deficiency in critically ill patients: highlighting the role of hepcidin. Crit Care. 2011;15:210.

36. Lindstrom E, Hossain MB, Lonnerdal B, Raqib R, Arifeen SE, et al. Prevalence of anemia and micronutrient deficiencies in early pregnancy in rural Bangladesh. Acta Obstet Gynecol Scand. 2011:90:47-56.

37. Haque M, Budi A, Azam Malik A, Suzanne Yamamoto S, Louis V, et al. Health coping strategies of the people vulnerable to climate change in a resourcepoor rural setting in Bangladesh. BMC Public Health. 2013;13:565.

38. Egereonu UU, Nwachukwu UL. Evaluation of the surface and groundwater resources of Efuru river catchment, Mbano, South Eastern Nigeria. Modelling, Measurement and Control. 2005. 66

39. Rahman AA, Ravenscroft P. Groundwater resources and development in Bangladesh: background to the arsenic crisis, agricultural potential and the environment. Dhaka: The University Press Limited; 2003. 466 p.

40. Rasheed S, Jahan S, Sharmin T, Hoque S, Khanam MA, et al. How much salt do adults consume in climate vulnerable coastal Bangladesh? BMC Public Health. 2014;14.

41. Khan AE, Scheelbeek PFD, Shilpi AB, Chan Q, Mojumder SK, et al. Salinity in drinking water and the risk of (pre)eclampsia and gestational hypertension in coastal Bangladesh: a case-control study. PLoS ONE. 2014:9:e108715.

42. Brown IJ, Tzoulaki I, Candeias V, Elliott P. Salt intakes around the world: implications for public health. Int J Epidemiol. 2009;38:791-813.

43. World Health Organization (WHO). Population salt reduction strategies for the prevention and control of non-communicable diseases in South-East Asia region. New Delhi: World Health Organization; 2013. p. 48.

44. He FJ, Li J, MacGregor GA. Effect of longer term modest salt reduction on blood pressure: Cochrane systematic review and meta-analysis of randomized trials. BMJ. 2013;346.

45. Saint-Jacques N, Parker L, Brown P, Dummer T. Arsenic in drinking water and urinary tract cancers: a systematic review of 30 years of epidemiological evidence. Environ Health. 2014;13:44

46. Hossain M, Rahman SN, Bhattacharya P, Jacks G, Saha R, et al. Sustainability of arsenic mitigation interventions - an evaluation of different alternative safe drinking water options provided in MATLAB, an arsenic hot spot in Bangladesh. Front Environ Sci. 2015;3.

47. Santra SC, Samal AC, Bhattacharya P, Banerjee S, Biswas A, et al. Arsenic in food chain and community health risk: a study in gangetic west Bengal. Procedia Environ Sci. 2013;18:2-13.

48. Chapman D. Water quality assessment - a guide to use biota, sediment and water in environmental monitoring. Report No. 041921590. London: E\&FN Spon, an imprint of Chapman \& Hall; 1996. 626 p. 